\title{
EL REFLEJO DE LOS VALORES EDUCATIVOS DEL MOVIMIENTO OLÍMPICO EN EL CURRÍCULO DE EDUCACIÓN FÍSICA ESPAÑOL. ESTUDIO DE CASO DEL CENTRO DOCENTE SANTA ANA Y SAN RAFAEL EN LA COMUNIDAD DE MADRID
}

\author{
$D^{a}$. Natalia Ventola López \\ natii1993@hotmail.com \\ Dra. Ma Eugenia Martínez-Gorroño \\ eugenia.martinez@uam.es \\ Universidad Autónoma de Madrid \\ Fecha de recepción: Febrero 2016 \\ Fecha de aceptación: Septiembre 2016 \\ http://dx.doi.org/10.15366/citius2016.9.2.004
}

\section{Resumen:}

El trabajo de investigación llevado a cabo ha tenido como objetivo principal identificar la presencia de los valores educativos del ideario olímpico en la realidad educativa de la Educación Secundaria que en la actualidad se imparte en España. A partir de la vigencia de la Ley Orgánica de Educación española (LOE) del 2006 en el área de Educación Física de la Educación Secundaria Obligatoria y concretamente a partir del estudio del caso en la realidad de un Centro Educativo de la Comunidad de Madrid, "el Colegio Santa Ana y San Rafael". En el trabajo se ha profundizado en la labor profesional del docente especialista del área y sus resultados relativos a la comunicación del ideario olímpico al alumnado.

Palabras clave: Valores, Principios Olímpicos Fundamentales, Educación Física, Movimiento Olímpico.

Title: THE REFLECTION OF EDUCATIONAL VALUES OF OLYMPIC MOVEMENT IN THE SPANISH PHYSICAL EDUCATION CURRÍCULUM. A CASE STUDY OF THE SANTA ANA AND SAN RAPHAEL SCHOOL IN THE COMMUNITY OF MADRID.

\begin{abstract}
:
The purpose of this study is to identify the presence of Olympic values and ideals in current Spanish Secondary education, since the introduction of the Organic Law of Education (LOE) 2006, in Physical Education. Looking at Secondary Obligatory Education generally and Santa Ana and San Raphael specifically and delving into the work of the P.E specialist in his area and his results relating to the application of the Olympic ideals.
\end{abstract}

Key words: Values, Olympic fundamental principles, Physical Education, Olympic Movement.

\section{Introducción y objetivos}

El presente trabajo parte de nuestra vinculación con el ideario olímpico, del interés por conocer la realidad de la práctica docente y por saber si en la materia de Educación Física, y especialmente en los contenidos relativos al deporte, se transmiten los valores que contiene el ideario olímpico, ya que el desarrollo de valores en el proceso educativo nos parece de vital importancia, especialmente como consecuencia de las circunstancias en las que la sociedad de consumo actual se desenvuelve. 
Como es sabido, el deporte forma parte de los contenidos que recoge la actual ley educativa española. Ya, en el apartado de "competencias básicas" en el Real Decreto 1631 del 2006 se hace alusión a aspectos de carácter actitudinal, por ello, los contenidos deportivos se nos muestran como una oportunidad para transmitir los valores que implica la filosofía del olimpismo. Por otra parte, teniendo en cuenta la popularidad que hoy alcanza el fenómeno social que son los Juegos Olímpicos, pensamos que la utilización de su ideario, puede ser especialmente idónea para que sus valores puedan encontrar un cauce de conexión que favorezca su acogida por parte de los estudiantes.

Por tanto, en este trabajo nos planteamos como objetivo principal conocer e identificar la presencia de los valores educativos del ideario olímpico en la realidad educativa de la Educación Secundaria que en la actualidad se imparte en España, a partir de la vigencia de la Ley Orgánica de Educación Española (LOE) del 2006 en el área de Educación Física de la Educación Secundaria Obligatoria. Teniendo en cuenta las circunstancias del comienzo de este trabajo monográfico y las limitadas posibilidades, nos planteamos un estudio de caso centrado en la realidad de un centro educativo de la Comunidad de Madrid: el "Colegio Santa Anay San Rafael", profundizando en la labor profesional de un docente especialista del área, a fin de conocer y analizar los resultados relativos a la comunicación del ideario olímpico a su alumnado.

En base a este objetivo principal decidimos centrar nuestro trabajo en los tres objetivos específicos que a continuación se detallan, a fin de diseñar un esquema de trabajo coherente con los propósitos que previamente nos habíamos marcado:

A. Identificar y extraer de la Carta Olímpica Internacional del 2015 y de la vigente Ley Orgánica de Educación española (LOE) del 2006 en el área de Educación Física los contenidos comunes relativos a valores y actitudes, buscando el paralelismo entre el ideario del Movimiento Olímpico Moderno y los valores contenidos explícitamente en el curriculum de Educación Física.

B. Examinar y registrar las posibles coincidencias de los resultados obtenidos fruto del objetivo anterior (los valores comunes contenidos en el ideario olímpico y en la LOE) que puedan estar recogidos en el Proyecto Educativo del Centro (PEC) objeto de estudio, es decir, el colegio "Santa Ana y San Rafael" ubicado en la Comunidad de Madrid.

C. Conocer la presencia, valoración y utilización de los valores del Ideario Olímpico en la labor profesional del docente de Educación Física y su asimilación por parte del alumnado.

\section{Metodología}

Como primer paso obligado en todo trabajo de investigación, "la aproximación al objeto de estudio" que supone un acercamiento más profundo al tema a investigar, nos llevó a la lectura del trabajo de María Teresa Calle Molina y Ma Eugenia Martínez Gorroño (2014) "Presencia o ausencia de los valores del Movimiento Olímpico en el currículo español de Educación Secundaria Obligatoria en Educación Física: un estudio de dos casos". Este trabajo, nos fue útil, como fuente secundaria, nos sirvió de orientación metodológica y nos aportó información sobre la existencia de algunas fuentes primarias que habrían de ser imprescindibles para la realización del trabajo que nos proponíamos llevar a cabo.

Así, en primer lugar, procedimos a realizar la identificación de los valores educativos que implica el ideario olímpico a partir del análisis documental de la Carta Olímpica Internacional (2016) y el trabajo de "Binder, D. (2007). Teaching values: an olympic education toolkit". Centramos nuestro análisis en estos documentos siguiendo las premisas establecidas por Best (1982, p.81) relativas a la "crítica interna y externa de la fuente" tras ser identificados por nosotros como fuentes primarias ya que se trataba de documentos que parten de la estructura del 
Movimiento Olímpico actual y por tanto, su contenido supone un manifiesto de los valores que el ideario olímpico pretende difundir a partir del instrumento que implica el deporte, practicado bajo sus premisas.

Posteriormente realizamos otro análisis documental del currículo de Educación Física que según establece la ley se imparte en la Educación Secundaria Obligatoria en España, a partir del contenido del Real Decreto 1631 de 2006, bajo la perspectiva de identificar la posible presencia de los valores que, formando parte del ideario olímpico, pudieran estar también presentes explícita o implícitamente en él. El enfoque del análisis documental de este documento legislativo, como ya hemos señalado, partió del resultado del primer análisis documental citado en el punto anterior, realizado sobre la Carta Olímpica (2016) y el trabajo de Binder, D. (2007). Nos centramos en seleccionar aquellos valores referentes del currículo de Educación Física de la Educación Secundaria Obligatoria española que son similares a los Principios de la Carta Olímpica Internacional y por tanto, así, poder conocer cuáles de los que conforman la base de esta filosofía están presentes también en el curriculum. Todo ello, como hemos dicho, para conocer de qué manera puede estar inmersa la filosofía del Olimpismo en el currículo de Educación Física de la Educación Secundaria Obligatoria Española.

Posteriormente realizamos un análisis del Proyecto Educativo del Centro "Santa Ana y San Rafael", centro concertado, que imparte todos los cursos del nivel de Educación Secundaria obligatoria y que está ubicado en la Comunidad de Madrid. El Proyecto Educativo (PEC), es un documento que, según establece la legislación educativa española, debe estar depositado en los archivos de todos los centros docentes, y está elaborado por la Comunidad Educativa correspondiente. En él están reflejadas las directrices que marcan su actividad docente y se recoge el ideario y los principios que rigen y dan personalidad propia al centro. Nos fue permitido consultar el contenido del citado PEC tras ser efectuada la adecuada petición al equipo directivo del citado colegio. Su análisis documental fue realizado desde la misma perspectiva antes mencionada, relativa a la identificación de la posible presencia de los valores que implica el ideario olímpico.

Con el resultado del trabajo de los análisis documentales, mencionados en los párrafos precedentes, procedimos a hacer una aproximación a la perspectiva que sobre los valores objeto de estudio pudiera tener el profesor de Educación Física del centro estudiado: Alan John Orr. Se trataba de conocer el enfoque y la disposición que, con respecto a nuestro objeto de estudio, marcaba el devenir profesional del profesor especialista de Educación Física del centro investigado. Este aspecto cobraba una importancia fundamental y decisiva para alcanzar el éxito en el conocimiento que nos habíamos propuesto, ya que él era la persona responsable de llevar a la práctica todo el contenido teórico expuesto en el currículo de Educación Física de Educación Secundaria Obligatoria y en las directrices marcadas en el PEC.

Para ello, utilizamos la técnica de la entrevista, que abordamos como una "entrevista semiestructurada de final abierto" siguiendo los criterios de Hammer y Wildasky, 1990. Igualmente, todo su desarrollo estuvo marcado por todas las directrices establecidas por el diseño metodológico que, para la investigación, se llevan a cabo como forma habitual de trabajo en el Centro de Estudios Olímpicos de la UAM, y concretamente para la investigación que se realiza utilizando Fuentes Orales. Directrices que ya son habituales en los trabajos de investigación para los TFMs y en los trabajos del programa de doctorado que se encuadran en el citado CEO de la UAM. Así, por ejemplo, procedimos a la elaboración de un guión que estuviera configurado en base a los objetivos que nos habíamos propuesto; procedimos al cuidado de los preliminares de acercamiento de la fuente oral al tema de investigación y a su interés; organizamos un primer contacto previo a la entrevista o tramitamos el permiso de autorización de grabación previo que según Comité de Ética de la Investigación de la UAM (aprobado por el Consejo de Gobierno del 
8 de febrero de 2013), se propuso al profesor previamente a la celebración de la entrevista y su grabación. Estos fueron varios aspectos, entre muchos otros que realizamos, marcados, como hemos dicho, por las ya citadas directrices.

Siguiendo las pautas metodológicas marcadas por Best (1982, p.81) llevamos a cabo la "crítica externa" de la fuente oral, seleccionando a Alan John por ser el docente responsable de la E.F. del nivel de secundaria. El favoreció en todo momento nuestra labor pudiendo así plantearnos y reflexionar sobre muchos de los aspectos observados en las clases y a su vez conversar sobre estos. Sin embargo, con respecto a la "crítica interna" (Ibídem) del contenido, debemos mencionar el breve testimonio que fue el resultado de la entrevista ya mencionada. Por ello, a fin de completar el acercamiento a la realidad de la presencia de los valores del ideario olímpico en las clases de E.F. del centro objeto de estudio, procedimos a completar metodológicamente nuestra investigación llevando a cabo "observaciones no participativas" de las clases durante la impartición de las sesiones que llevaba a cabo el citado profesor, a fin de darle el rigor metodológico adecuado a nuestro trabajo.

Para conocer el nivel de la presencia de los valores objeto de estudio en las clases de E.F., también utilizamos como técnica el cuestionario para la recogida de datos a fin también de contar con aspectos cuantitativos en los resultados. El cuestionario consta de preguntas cerradas con respuestas de opción múltiple. Se trata de un cuestionario de percepción de los Valores Olímpicos extraído de Preuss, H; Schütte, N; Könecke, T; DaCosta, L. (2014) en Olympic Ideals as seen by Olympic Scholars and Experts. La elección de esta herramienta estuvo condicionada por la circunstancia de tratarse de un cuestionario que ya ha sido utilizado por otros investigadores con similares objetivos y por el hecho de que en sus contenidos han participado también diferentes estructuras de los Centros de Estudios Olímpicos de varios países.

El cuestionario fue presentado para su cumplimentación a dos grupos distintos de participantes. Por un lado, el primer grupo, conformado solo por el docente de Educación Física del nivel de Educación Secundaria del centro objeto de estudio: Alan John Orr, ya mencionado. Por el otro, sus alumnos, que conformaban un grupo mucho más numeroso, pero que obviamente suponía un tipo diferente de participante.

Finalmente, siguiendo las directrices marcadas, procedimos a llevar a cabo la triangulación de los datos obtenidos fruto de los análisis documentales, de la entrevista, de los cuestionarios y de la "observación no participativa" que nos permitió el profesor. Con todo ello, procedimos a elaborar y redactar los resultados y finalizamos con la redacción de las conclusiones.

\section{3.- Primeros resultados}

A partir del análisis documental realizado con las fuentes primarias ya citadas: la Carta Olímpica Internacional, (2006) y el trabajo "Teaching values: an olympic education toolkit" (Binder, 2007) los valores integrados dentro del ideario olímpico identificados fueron:

I. La alegría del esfuerzo. El deporte realizado por los jóvenes debe desarrollar habilidades que provoquen un cambio en ellos mismos, ya no sólo físicamente, sino intelectualmente y en la conducta.

II. Fair play (Juego limpio). El juego limpio puede ser aplicado a muchas situaciones de la vida diaria, es por ello, que es fundamental aprender una conducta de juego limpio en el deporte, ya que supone un refuerzo en el desarrollo de la conducta, en la comunidad y en la vida. 
III. Respeto hacia los demás. Es imprescindible aprender a vivir en un ambiente multicultural, respetando la diversidad y promoviendo un entendimiento internacional para propiciar el estableciendo de una conducta pacífica. El deporte desarrollado a partir de los Principios Olímpicos pretende promover el respeto y la paz.

IV. Búsqueda de la excelencia. Proyectar a los jóvenes hacia la búsqueda de la excelencia es fundamental para que su actitud vital sea positiva y para potenciar su capacidad y deseos de superación. El deporte es un instrumento idóneo para identificar y establecer los propios retos que se desean conseguir.

V. Equilibrio entre cuerpo, mente y voluntad. Es trascendental aprender a tomar conciencia de la propia persona en su globalidad. Es importante llegar a conocer que a través del movimiento se desarrollan todas las capacidades y potencialidades humanas identificadas con el cuerpo, el espíritu o la mente.

Tras realizar el análisis documental del currículo de Educación Física de la Educación Secundaria Obligatoria vigente, ya citado y proceder a su comparación con los valores presentes en el ideario olímpico, obtuvimos los siguientes resultados:

En primer lugar, tras analizar los diez objetivos generales, sólo uno de todos ellos estaba relacionado con dos valores de la Carta Olímpica que en este caso son el "respeto hacia los demás" y el "juego limpio".

Fue en el objetivo número 8 , en el que encontramos presente ya en el mismo enunciado del objetivo la deportividad o juego limpio, que se hacía constar textualmente como: "Mostrar habilidades y actitudes sociales de respeto, trabajo en equipo y deportividad en la participación en actividades, juegos y deportes, independientemente de las diferencias culturales, sociales y de habilidad".

Posteriormente, tras un análisis del citado currículum curso por curso, se obtuvieron los siguientes resultados:

En $1^{\circ}$ de la ESO, destacaron los valores tanto de "respeto" como de "juego limpio". Ya que, en el bloque de contenidos de "Condición física y salud" encontramos la siguiente cita textual: "Respeto y aceptación de las reglas de las actividades, juegos y deportes practicados". Concepto que estimamos relacionado directamente con el valor identificado en el contenido de la Carta Olímpica como "juego limpio”.

Y, por último, en el bloque de "Actividades en el medio natural", se encuentra el siguiente párrafo: "Aceptación y respeto de las normas para la conservación del medio urbano y natural". Por tanto, de nuevo, encontramos presente el respeto como valor educativo, aunque este no esté relacionado directamente con las personas, sino relativo al entorno, por lo que igualmente estimamos que sí está presente en cuanto a lo que se entiende como valor.

En $2^{\circ}$ de la ESO, identificamos en los contenidos del bloque de "Juegos y deportes" y de "Expresión corporal", y en un "criterio de evaluación", varios apartados que se relacionan directamente con los valores identificados en el Ideario Olímpico. Estos valores son: el respeto hacia los demás y el juego limpio.

Sin embargo, en $3^{\circ}$ de la ESO no hemos podido identificar ningún valor que tuviera especial relación con los valores del ideario olímpico. 
En $4^{\circ}$ de la ESO, solo pudimos extraer un contenido relacionado con los valores anteriormente citados. Ya que, desde nuestro punto de vista, este contenido abarca tanto el respeto como el juego limpio.

Las siguientes tablas recogen los datos de los resultados y la interrelación de los valores contenidos en los documentos analizados:

Tabla 1.

Fair Play (Juego Limpio)

\begin{tabular}{|c|c|}
\hline \multirow{5}{*}{ FAIR PLAY (JUEGO LIMPIO) } & $\begin{array}{l}\text { "Respeto y aceptación de las reglas de las } \\
\text { actividades, juegos y deportes practicados". } \\
\left.\text { (Contenido } 1^{\circ} \mathrm{ESO}\right) \text {. }\end{array}$ \\
\hline & $\begin{array}{l}\text { "Respeto y aceptación de las normas de los } \\
\text { deportes de adversario y de las establecidas por el } \\
\text { grupo". (Contenido } 2^{\circ} \mathrm{ESO} \text { ). }\end{array}$ \\
\hline & $\begin{array}{l}\text { "Autocontrol ante las situaciones de contacto } \\
\text { físico que se dan en los juegos y el deporte". } \\
\left.\text { (Contenido } 2^{\circ} \mathrm{ESO}\right) \text {. }\end{array}$ \\
\hline & $\begin{array}{l}\text { "Tolerancia y deportividad por encima de la } \\
\text { búsqueda desmedida de los resultados". } \\
\left.\text { (Contenido } 2^{\circ} \mathrm{ESO}\right) \text {. }\end{array}$ \\
\hline & $\begin{array}{l}\text { "Aceptación de las normas sociales y democráticas } \\
\text { que rigen en un trabajo en equipo" (Contenido } \\
4^{\circ} \mathrm{ESO} \text { ). }\end{array}$ \\
\hline
\end{tabular}

Tabla 2.

Respeto hacia los demás

\begin{tabular}{|l|l|}
\hline & "Mostrar habilidades y actitudes sociales de \\
respeto, trabajo en equipo y deportividad en la \\
participación en actividades, juegos y deportes, \\
independientemente de las diferencias culturales, \\
sociales y de habilidad" (Objetivo general, número \\
8 ).
\end{tabular}


En la fase siguiente, y tras analizar el contenido del documento que supone el Proyecto Educativo del Centro (PEC), los resultados mostraron, en primer lugar, que los tres valores más destacados y relacionados con el ideario olímpico fueron: el respeto, la búsqueda de la excelencia y el equilibrio entre mente, cuerpo y voluntad. Pero estos valores no estaban descritos ni definidos, solo citados en el PEC. Por ello, nos parece imprescindible hacer la reflexión de que es necesario conocer la forma en que se interpreten en la práctica para poder afirmar que sean similares o diferentes a la filosofía Olímpica. Como consecuencia, en esta fase del trabajo reflexionamos en cuanto a las carencias que ofrece el análisis documental y nos planteamos la necesidad imprescindible de consultar la interpretación de la fuente oral, en este caso, el docente de Educación Física.

\section{El trabajo con la fuente oral}

Por último, se realizó la entrevista al docente, ya citada bajo el modelo semiestructurado de final abierto (Hammer y Wildasky, 1990), ya mencionado. Su guión se estructuró organizado por categorías marcadas por los valores ya identificados, para intentar que no se produjeran lagunas. Por otra parte, decidimos introducir una pregunta dentro de una categoría para poder establecer comparaciones con la información obtenida por Binder en su trabajo "Teaching values", a fin de que los resultados nos pudieran dar una base sólida en el proceso metodológico de triangulación ya mencionado.

Tras recoger el testimonio prestado por la fuente oral y su transcripción, los resultados estuvieron marcados por un planteamiento dual desde la primera perspectiva teórica hasta la reflexión sobre la realidad de la práctica. Así, el docente en principio no destacó ni dio prioridad teóricamente a ninguno de los valores en la primera parte de la entrevista, pues según sus propias afirmaciones, para él todos tenían la misma importancia. Sin embargo, más adelante, reconoció que, en la práctica, la alegría del esfuerzo, para él destacaba por encima del resto, aunque también le dio mucha importancia al fair play. No obstante, observamos que a la búsqueda de la excelencia le otorgó menor valor. Por ello, y tras triangular la información obtenida a través de los diferentes recursos metodológicos ya mencionados en los apartados precedentes, estimamos que este último valor, por ejemplo, no es impartido en sus clases de la misma manera o simplemente no es interpretado de la forma en que en la filosofía olímpica se entiende.

Además de conocer la opinión del docente, como ya hemos mencionado en apartados anteriores nos pareció interesante conocer el nivel de asimilación de los valores de la filosofía Olímpica por parte del alumnado y así obtener datos que sirvieran para llevar a cabo una triangulación con los resultados obtenidos a partir de las otras fuentes y que permitieran elaborar unas conclusiones más completas.

\subsection{Resultados del cuestionario}

La primera parte del cuestionario, se orientó al que considerábamos como principal objetivo centrado en conocer el conocimiento de los estudiantes sobre la filosofía Olímpica y averiguar la forma en que ellos la asimilaban.

Con respecto a la primera pregunta: ¿Qué valores consideras importantes en la práctica deportiva?, la respuesta mayoritaria (90\%) fue: juego limpio, esfuerzo, amistad y respeto. Las respuestas de estos valores fueron permanentes, sin apenas diferencias entre género y edad.

Las respuestas a la segunda pregunta: ¿Cuáles de estos valores citados anteriormente forman parte del Ideario Olímpico?, tuvieron el más común denominador que dejaba patente el desconocimiento de la fílosofía Olímpica y, por tanto, de los valores que la conforman. 
Preguntados sobre el lugar dónde aprendieron los valores que actualmente llevaban a la práctica en sus actividades deportivas; el $70 \%$ de los estudiantes mostraron su ignorancia sobre su origen. Sólo el 10\% de estos admitieron que el colegio o el contenido impartido en Educación Física eran los principales responsables del origen de sus valores. Otro $10 \%$ de los estudiantes afirmaron que estos valores los aprendieron en sus clubes deportivos y, por último, el otro $10 \%$ afirmaron que los aprendieron en sus casas.

Los resultados estadísticos de la segunda parte del cuestionario, orientada a conocer el grado de importancia que los estudiantes otorgaban a los diferentes valores que se explicitaban en el cuestionario, fueron:

El valor más importante elegido por los estudiantes fue "fair play". El $78.3 \%$ de los estudiantes lo señalaron como de "alta importancia" y sólo el $0.9 \%$ afirmaron que no era importante.

Sin embargo, el valor equilibrio entre el cuerpo, voluntad y mente fue el menos valorado por los estudiantes; ellos se decantaron por la respuesta "no importante" en una alta proporción.

En relación al género, no existieron diferencias sustanciales, el "fair play" fue el valor más importante; el $44 \%$ de los chicos y el $46 \%$ de las chicas afirmaron esto, por lo tanto, fueron resultados muy similares.

En relación a la edad, existieron algunas diferencias en las respuestas. Los estudiantes de los 12-13 años dieron mayor importancia a la igualdad de oportunidades en la práctica deportiva sin discriminación. Sin embargo, los estudiantes de 14-15 y 16-17 años otorgaron mayor importancia al valor de "la alegría del esfuerzo".

\section{Conclusiones}

Podemos afirmar que el contenido relativo a los valores que supone el ideario olímpico es desconocido como tal por el alumnado del Colegio "Santa Ana y San Rafael" de la Comunidad Autónoma de Madrid.

El fair play es el valor que, aunque como los demás no fue identificado como parte del ideario olímpico, podemos afirmar que, como valor en la práctica deportiva, sobresale especialmente por su presencia en la realidad teórica y práctica del Colegio "Santa Ana y San Rafael" de la Comunidad Autónoma de Madrid. Estimamos que aparece explícitamente mencionado en su PEC cuando se hace referencia "al respeto" (en el que entendemos tanto a la normativa como a las personas). Fue uno de los valores que en nuestras observaciones "no participativas" realizadas en las sesiones de clase, pudimos comprobar que era uno de los más trabajados por el profesor diariamente en las clases de Educación Física. Fue mencionado también explícitamente por el docente en su testimonio y ofreció los datos cuantitativos más elevados en las respuestas del alumnado.

La "alegría del esfuerzo" es el segundo valor más considerado, que encabeza las prioridades que explícitamente afirma el docente tener, además de encontrarle especialmente presente durante las clases de Educación Física y ser el más valorado entre los alumnos más mayores, que sitúan en tercer lugar a la "igualdad de oportunidades".

El "equilibrio entre el cuerpo, voluntad y mente" si bien figura en el PEC del Colegio "Santa Ana y San Rafael" no fue señalado de forma especial en el testimonio del docente y no nos pareció percibir ninguna alusión al mismo en las observaciones de las clases. Fue valorado 
como "no importante" por parte del alumnado. Por lo tanto, estimamos que este valor, que supone una aportación definitiva de la filosofía Olímpica, es una debilidad a destacar en la realidad práctica de la cotidianeidad educativa del colegio objeto de estudio, y en el que se precisa incidir especialmente.

Si bien la "solidaridad" no aparece explícitamente citada en su PEC y no fue un aspecto especialmente mencionado en el testimonio del docente, la solidaridad fue un valor que pudimos observar en la cotidianeidad de las actividades promovidas en el Colegio diariamente, no sólo durante el horario lectivo, sino siendo protagonista en las actividades extraescolares.

La búsqueda de la excelencia es un objetivo del ideario olímpico que no fue identificado como valor importante por parte del docente, aunque si se encontró presente en el PEC, tampoco fue considerado importante por parte del alumnado.

\section{FUENTES Y BIBLIOGRAFÍA}

\subsection{Fuentes primarias}

- Comité Olímpico Internacional (2015). Olympic Charter. Lausanne Switzerland. Consultado el 12/02/2016 en https://www.um.es/documents/933331/0/CartaOlimpica.pdf/8c3b36b211a2-4a77-876a-41ae33c4a02b

- Real Decreto 1631/2006, de 29 de diciembre, por el que se establecen las enseñanzas mínimas correspondientes a la Educación Secundaria Obligatoria. Boletín Oficial del Estado, 5, 677773.

\subsubsection{Fuente oral}

- Alan John Orr, profesor de Educación Física del Colegio Santa Ana y San Rafael de la Comunidad Autónoma de Madrid. Entrevista realizada en el mes de mayo del 2016.

\subsection{Bibliografía}

- Andreu, E. (2010). Olympic values: the end does not justify the means. Journal of Human Sport and Exercise. Universidad de Alicante, 5, 15-23. http://dx.doi.org/10.4100/jhse.2010.51.03

- Arufe Giráldez, F. (2011). La educación en valores en el aula de educación física. ¿Mito o realidad? Revista digital de Educación Física. 2, 9. Recuperado de: http://emasf.webcindario.com/La_educacion_en_valores_en_el_aula de EF.pdf

- Binder, D. (2007). Teaching values: an Olympic Education toolkit. A Project of the International Olympic Committe. Laussanne Switzerland.

- Binder, D. (2010). Olimpismo en las escuelas: la Educación Olímpica como centro de la educación en valores. Lecciones universitarias olímpicas. Barcelona: Centre d'Estudis Olímpics (UAB). Cátedra Internacional de Olimpismo (CIO-UAB). Recuperado de:

http://olympicstudies.uab.es/2010/docs/binder_spa.pdf 
- Calle Molina, M. y Martínez Gorroño, M. E. (2014). Presencia o ausencia de los valores del Movimiento Olímpico en el currículo español de Educación Secundaria Obligatoria en educación física: Un estudio de dos casos. Citius, Altius, Fortius. Humanismo, sociedad y Deporte: Investigaciones y ensayos, 7(1), 99-121. Centro de Estudios Olímpicos de la Universidad Autónoma de Madrid. Recuperado de

http://cdeporte.rediris.es/revcaf/Numeros\%20de\%20revista/Vol\%207\%20n1/Vol7_n1_Call e_Martinez.pdf

- Anaya Maya, C. (2014). Transformación social a través del deporte y los valores olímpicos. Medellín-Colombia, un caso de estudio. Citius, Altius, Fortius. Humanismo, sociedad y Deporte: Investigaciones y ensayos, 7(2), 23-24. Centro de Estudios Olímpicos de la Universidad Autónoma de Madrid. Recuperado de http://cdeporte.rediris.es/revcaf/Numeros\%20de $\% 20$ revista/Vol7\%20n2/Vol7_n2_AmayaN aya.pdf

- Gutiérrez Sanmartín, M. (1995). Valores sociales y deporte. Madrid: Gymnos.

- Gutiérrez Sanmartín, M. (2003). Manual sobre valores en la educación física y el deporte. Barcelona: Paidós.

- Gutiérrez Sanmartín, M. (1991). Desarrollo de los valores en la educación física y el deporte. Apunts.51, 100-108.

- Hammer, D. y Wildavsky, A. (1990). La entrevista semi-estructurada de final abierto. Aproximación a una guía operativa. Historia y Fuente Oral, 4, $23-61$.

- Harerimana, T. (2014). Los valores Olímpicosen la educación: análisis, reflexiones y recomendaciones. Citius, Altius, Fortius. Humanismo, sociedad y Deporte: Investigaciones y ensayos, 7 (1), 61-65. Madrid: Centro de Estudios Olímpicos de la Universidad Autónoma de Madrid. Recuperado de http://cdeporte.rediris.es/revcaf/Numeros\%20de\%20revista/Vol\%207\%20n1/Vol7_n1_Hare rimana.pdf

- Molina, J. M. (2011). Visión del Olimpismo y sus repercusiones educativas entre universitarios de Magisterio de Educación Física, Grado de la Actividad Física y el Deporte de la Región de Murcia. Tesis doctoral. Universidad Católica de Murcia.

- Müller, N. (2004). Educación Olímpica.Lección universitaria olímpica. Barcelona: Centre dÉstudis Olímpics (UAB). Cátedra Internacional de Olimpismo (CIO-UAB).

Recuperado de: http://olympicstudies.uab.es/lectures/web/pdf/spa_muller.pdf

- Nanayakkara, S. (2012). Olimpism in practice: an evaluation of the effectivenessof an Olympism education programme to resolve conflicts between primary school students in Sri Lanka, Degree of Doctor of Philosophy. Thesis. University of Canterbury.

- Pena Pérez, X. (2015). Los valores olímpicos en la escuela. En V. Arufe Giráldez (Coord.). $V$ Congreso Mundial del Deporte Escolar, A Coruña, 5-7 noviembre 2015 (pp.1-16). A Coruña: Sportis.

- Robles, E. (2013). Juegos Olímpicos: una propuesta didáctica e interdisciplinar. Revista digital de Educación Física. 22, 72-91. 
- Preuss, H., Schütte, N., Könecke, T. y Lamartine, D. (2014). Olympic ideals as seen by Olympic Scholars and Experts. Johannes Gutenberg- University Mainz.

- Zapata, M. (2006). La educación Olímpica en las escuelas. Seminarios sobre los valores olímpicos como objeto de investigación en el campo de la educación y la cultura en España y Brasil. Abril. (pp.197-209) Barcelona: Centre d’Éstudis Olimpics. 